\title{
Regression-contingent analyses of eye movements during sentence processing: Reply to Rayner and Sereno
}

\author{
GERRY T. M. ALTMANN \\ University of Sussex, Brighton, England
}

\begin{abstract}
Altmann, Garnham, and Dennis (1992) explored contextual influences on syntactic ambiguity resolution by monitoring eye movements during reading. In order to resolve a conflict of interpretation given that the different eye movement measures yielded different patterns, we introduced a regression-contingent analysis of reading times, separating trials according to whether the eyes departed from the region of interest with a leftward (regressive) or rightward movement. Rayner and Sereno (1994) argue that various assumptions which they claim underlie the motivation for introducing the regression-contingent measure are in fact flawed. In this paper I demonstrate that these assumptions are incorrectly ascribed to us (while agreeing that they are incorrect), and that Rayner and Sereno's re-analysis of an earlier study by Rayner, Garrod, and Perfetti (1992) neither questions nor threatens the generalizability of the regression-contingent measure. Finally, I discuss some of the uncertainties surrounding the interpretation of first-pass reading times which further motivate the measure we adopted in Altmann et al.'s (1992) study.
\end{abstract}

Rayner and Sereno (1994) have made a number of points regarding an earlier paper by Altmann, Garnham, and Dennis (1992). In that paper, we were concerned with whether contextual information can influence the parser's initial choice of analysis in the face of a local syntactic ambiguity. We compared locally ambiguous structures such as Sentence 1 below with unambiguous controls such as Sentence 2:

1. He told the woman that he'd risked his life for to install a smoke detector.

2. He asked the woman that he'd risked his life for to install a smoke deector.

In the absence of any explicit context (the "null" context condition), a garden path effect was implicated in Sentence 1 by increased first-pass reading times per character ${ }^{1}$ in the disambiguating (italicized) region, and more firstpass regressions (leftward movements) out of this region. However, when these sentences were preceded by referentially felicitous contexts (Altmann, 1988; Altmann \& Steedman, 1988; Crain \& Steedman, 1985; Steedman \&

The research described here was supported by SERC/MRC/ESRC Grant SPG8920151 awarded to myself and Alan Garnham. My thanks to Alan Garnham, Yvette Dennis, and Judith Henstra for their involvement in the research. In addition, I thank Margaret Jean Intons-Peterson for inviting me to respond to the paper by Rayner and Sereno, and Keith Rayner and an anonymous reviewer for comments on an earlier version of this response. Requests for reprints should be addressed to G. T. M. Altmann, Laboratory of Experimental Psychology, University of Sussex, Brighton BN1 9QG, England.

-Accepted by previous editor, Margaret Jean Intons-Peterson
Altmann, 1989), a conflict arose because although there was now no difference in first-pass-per-character reading time in the critical region (29.9 vs. $29.2 \mathrm{msec}$ for the experimental and control sentences, respectively), there was a difference in the probability of making a first-pass regression out of that region (0.34 vs. 0.21$)$.

To reconcile these apparently conflicting data, we introduced a regression-contingent analysis of first-pass reading times-we separated the first-pass reading times according to whether or not a leftward regression had been made out of the region in which first-pass reading times were being calculated. Our rationale for doing this was simple: If a garden path occurred, and if this did not result in a regression, then we could expect the garden path to manifest itself as an increase in the time taken to read the region (cf. Frazier \& Rayner, 1982, who suggested that readers can perform reanalysis following a garden path without having to move their eyes back through the text). In the null context, we found that reading times in the absence of a regression were larger in the disambiguating region of the experimental sentence $(46.9 \mathrm{msec})$ than in the corresponding region of the control $(35.1 \mathrm{msec})$. In the felicitous context condition, this difference disappeared ( 31.0 vs. $30.2 \mathrm{msec}$ ). We argued on the basis of this result and the fact that these data represented the majority of the trials ( $71 \%$ in Experiment 1, from which the data above are taken, and $76 \%$ in Experiment 2) that contextual information prevented a garden path effect that would otherwise be predicted by the garden path theory of sentence processing (cf. Ferreira \& Clifton, 1986; Rayner, Carlson, \& Frazier, 1983).

One crucial assumption was made in that study: that if the garden path effect had not been prevented in the 
felicitous contexts, we would have seen a pattern more similar to the null context reading times-namely, an increase in reading times in the absence of a regression in the disambiguating region relative to the control. If, for instance, the felicitous context simply eliminates some proportion of regressions, but otherwise does not eliminate the garden path, then we would have expected some reading time evidence of the garden path on trials without regressions (cf. the null context pattern).

Rayner and Sereno (1994) make a number of distinct claims in their critique of Altmann et al. (1992): that we have assumed that syntactic garden paths necessarily result in regressive eye movements; that data reported by Rayner, Garrod, and Perfetti (1992) are ideal for testing the generalizability of the regression-contingent measure, and that according to our argument, the garden path effects that were observed in Rayner et al.'s (1992) study should be eliminated or weakened when trials with regressions are eliminated; that contrary to "our" argument, the garden path effect was inexplicably stronger when trials with regressions were eliminated from the analysis; and that the differences between the studies may be due to memory span differences (cf. Just \& Carpenter, 1992) between subjects, and that a recent study by Altmann, Garnham, and Henstra (1994) may reflect on this issue. I shall consider each of these claims in turn before providing additional motivation for the regression-contingent analysis of eye movements.

\section{Equating Syntactic Garden Paths With Regressive Eye Movements}

We (Altmann et al., 1992) do not believe that "regressive eye movements are a necessary consequence of subjects' being garden pathed," as is indicated by our previous statements:

If a garden path occurs on the majority of trials, but regressions only occur on a minority of trials, then we must expect evidence of a garden path even when there has been no regression. (Altmann et al., 1992, p. 695)

A regression from the disambiguating region is not evidence per se of a garden path. (p. 697, n. 7)

Our hypothesis about target sentences presented in felicitous contexts was that garden paths occurred in only a small number of cases (discussed more fully in that paper), and that any reading time evidence for those garden paths (as found in Experiment 2) could be accounted for by the small number of occasions on which a regression occurred. This does not mean that we assumed that a garden path necessarily resulted in a regression.

\section{Generalizing From the Regression-Contingent Analysis to Rayner et al.'s (1992) Study}

We suggested in the 1992 paper that noncontingent firstpass reading times could possibly hide any disambiguating effects of nonsyntactic factors on the initial stages of syntactic ambiguity resolution. Rayner et al. (1992) failed to find nonsyntactic effects on the initial analysis, and Rayner and Sereno (1994) therefore reanalyzed the data to see whether a contingent analysis would yield evidence of nonsyntactic influences.

Rayner and Sereno (1994) add that "If regressions are a necessary consequence of garden pathing, then, when a regression-contingent analysis (in which trials with regressions are eliminated from the data set) is performed on Rayner et al.'s (1992) data, the garden path effect should be eliminated or considerably weakened.' This prediction is valid if regressions are a necessary component of garden pathing. However, it is incorrect to attribute this assumption, as they do, to our 1992 argument.

At issue, then, is what happens when Rayner et al.'s (1992) data are analyzed with Altmann et al.'s (1992) procedure. In the analysis that Rayner and Sereno (1994) report in the main body of their paper, they did not in fact employ the same procedure as our own, although they report in an endnote (note 5) that they did carry out our procedure, and that it yielded the same pattern as did the procedure they adopted in the main text. I argue in the next section that this pattern, indicating garden pathing, is unsurprising. However, some defense of our own procedure, against that favored by Rayner and Sereno, is warranted.

We had calculated reading times in a given region contingently upon whether or not a regressive movement had occurred out of that region: for instance, to calculate what we called "reading times in the absence of a regression" in the disambiguating region to install (see Sentence 1 above), we eliminated just those trials in which to install was left with a regressive eye movement. Rayner and Sereno (1994) reported data that were contingent on a regressive movement out of either that region or the region that followed (a smoke detector). They suggest that "if regressions are the only hallmark of being garden pathed, it makes sense to use the regression-contingent analysis on trials in which subjects regressed only after having reached the disambiguating region" (note 5). In fact, it is exactly because regressions are not the only hallmark of being garden pathed that we did not segregate reading times according to this method. Indeed, we made this same point in our own note 7 , where we explicitly considered this procedure.

According to Rayner and Sereno's (1994) own figures (note 6), in almost half the trials that were excluded in the no-regression analysis, the eyes did not leave the disambiguating region with a regressive movement-these trials were excluded because a regression had occurred in the following region (which in Rayner et al.'s, 1992, study constituted the final region, and which in our studies yielded the greatest numbers of regressions irrespective of whether a garden path had occurred or whether the sentence was locally ambiguous or not). Thus, Rayner and Sereno's procedure excluded far more trials (almost twice as many) than our own.

\section{On the Discrepancy Between Rayner et al.'s and Altmann et al.'s Studies}

Rayner and Sereno (1994) admit to having no ready explanation for why the garden path effect became stronger in Rayner et al.'s (1992) study when they excluded trials 
with regressions, whereas the effect was eliminated in Altmann et al.'s (1992) study following application of our own regression-contingent procedure.

In that study, we observed a garden path effect in the null context that amounted to $6.8 \mathrm{msec}$ per characterthe difference at the disambiguating region between the locally ambiguous relative and its control. When we eliminated trials in which a regressive eye movement occurred out of the region, this difference increased to $11.8 \mathrm{msec}$ per character. It would thus appear that there is considerable consistency across the studies: where there is a garden path, an analysis of reading times on trials without regressive eye movements will make that effect more pronounced. $^{2}$

A more important issue is why the regression-contingent measure should indicate a garden path effect in one study, and not in the other. One possibility, noted also by Rayner et al. (1992, p. 128), is that their contexts contained just one referent for the head of the noun phrase (the equivalent of the woman in the earlier examples), and in previous studies (Altmann \& Steedman, 1988; Crain \& Steedman, 1985), we had argued that such contexts needed to be contrasted with others containing more than one referent in order to yield evidence of contextual influences on initial parsing decisions. Thus, it is not surprising that Rayner et al. (1992) failed to overturn the garden path effects predicted by the minimal attachment strategy (Frazier, 1979) ${ }^{3}$ And again, the fact that the garden path effect was stronger when trials on which regressions occurred were eliminated, as was also the case in Altmann et al.'s (1992) study, perhaps attests to the generalizability of our regression-contingent analyses. ${ }^{4}$

In order to explain the discrepancy between Altmann et al.'s (1992) and Rayner et al.'s (1992) results, Rayner and Sereno (1994) ignore the differences just described. Instead, they offer an account based either on different reading strategies employed by the subjects in the different studies or on memory span differences between them (cf. Just \& Carpenter, 1992). They note that in a recent study of ours (Altmann et al., 1994), we did not make use of a regression-contingent analysis because readers regressed so infrequently. They suggest that memory span differences between the subjects in our two studies may have contributed to the different number of regressions, while acknowledging that structural differences between the targets in the two experiments may also have played a part.

The critical regions in the two studies are italicized in the examples below:

3. He told the woman that he'd risked his life for to install a smoke detector. (Altmann et al., 1992)

4. He told the woman that had been waiting for him that they were both very lucky. (Altmann et al., 1994)

In Sentence 3, the probability of making a first-pass regression out of to install in the null context was .44. The equivalent probability for that had been in Sentence 4 was .08. Given that the subjects in the two studies were drawn from the same population, we believe that the dearth of regressions in the region that had been owes to the fact that this region does not pose as severe a processing problem as does to install in the 1992 target. Minimal attachment predicts that the item that will initially be interpreted as a complementizer, and that this hypothesis has to be abandoned on encountering had been. A combination of the proximity of the disambiguating information to the ambiguous item itself and the associated fact that reanalysis simply involves reinterpreting a single lexical item may lead to relatively unproblematic reprocessing (although not so unproblematic that it does not show up on first pass reading times-see Altmann et al., 1994, for further details). Subjectively, at least, there is a big difference between the items in terms of their associated processing complexity. One does not need to appeal to memory span or strategic differences between the subjects in the two studies in order to explain the different patterns of regression.

\section{Regression-Contingent Analyses and First-Pass-per-Character Reading Times}

In addition to the rationale described both here and in Altmann et al. (1992), there is a further reason for adopting regression-contingent procedures-namely, that noncontingent per character reading times are subject to an artifact introduced by dividing the reading time for a region by the number of characters in that region.

The following is a (nonexhaustive) list of materials typical of eye-tracking experiments on garden path materials (only the versions of each sentence that violate the usual parsing preferences are shown here):

5. Since Jay always jogs a mile (and a half) seems like a very short distance to him. (Frazier \& Rayner, 1982)

6. The spy saw the cop with a revolver but the cop didn't see him. (Rayner et al., 1983)

7. The woman rushed to the hospital had given birth safely. (Britt, Perfetti, Garrod, \& Rayner, 1992; cf. Ferreira \& Clifton, 1986, and Rayner et al., 1992)

8. He told the woman that he'd risked his life for to install a smoke detector. (Altmann et al., 1992)

9. Peter read the books on the war instead of the other books.(Britt et al., 1992)

10. She decided to take the cheese from the farmer out of her bag to eat for their lunch. (Rayner et al., 1992)

11. He told the woman that had been waiting for him that they were both very lucky. (Altmann et al., 1994)

In each sentence, the italicized region represents the region in which first-pass reading times per character were calculated.

There are several reasons why the eyes might make a regressive movement out of a region (see Altmann et al., 1992; and Frazier \& Rayner, 1982; and Rayner, Sereno, Morris, Schmauder, \& Clifton, 1989, for further discussion), but crucially, exactly where from within the region 
the regression is initiated may not be taken into account when one is calculating per character reading times. For example, when the eyes travel through the entire italicized region of Sentence 6 above, the first-pass reading time for that region would be divided by 35 , the number of characters in the region. However, if the furthermost point that the eyes reached before regressing out of the region was the item revolver, it would not be appropriate to divide the time spent prior to the regression by the length of the entire region-the resulting first-pass per character reading time would have been calculated through the use of a denominator that did not reflect the number of characters read. And the more such trials, the more the noncontingent reading times will have been contaminated by, in effect, dividing the reading times on certain trials by an arbitrary number. ${ }^{5}$ Indeed, given that regressive eye movements can take place within a region, it is not clear whether any analysis in terms of per character reading times is free of the kinds of contaminant just described. ${ }^{6}$

A related issue is described by Rayner et al. (1989), who point out that in cases of early regressions out of a region, the first-pass reading time assigned to that region would be inappropriately short, simply because the time spent on a word later on in that region (i.e., subsequent to the regressive eye movement) would not be included in the first-pass reading time for that region (it would be included in the second-pass reading time). ${ }^{7}$ It would appear, then, on the basis of Rayner et al.'s (1989) point, and the per character problem, that the interpretation of first-pass reading times, whether per character or not, is far from straightforward. The regression-contingent measure has imperfections, but represents an attempt to acknowledge some of the problems inherent in the interpretation of eye movements.

\section{Conclusions}

Rayner and Sereno (1994) have made a number of claims regarding Altmann et al.'s (1992) study. I have argued, contrary to those claims, that we did not assume that garden paths necessarily result in regressive eye movements; that the reanalysis of Rayner et al.'s (1992) data is useful only insofar as it demonstrates that context effects were not hidden by the noncontingent reading times; and that it is quite unsurprising that context effects were not found to influence initial parsing decisions in that study.

Rayner and Sereno (1994) conclude their discussion of contextual influences on parsing decisions by suggesting that we (collectively) should adopt a strategy aimed at determining when contextual information influences the parser, not simply whether it does so (an issue that was addressed explicitly in Altmann et al., 1992, and Altmann et al., 1994). In recent work, I and my colleagues have attempted to do exactly this, but subject to the constraints imposed by the experimental tools available to us. Eye movements may well provide a finer window within which to view local processing difficulties, but the view through that window is far from clear. Only by establishing the distorting effects of different methodologies (including different eye movement measures) can we progress in our understanding of the influences on syntactic parsing decisions.

\section{REFERENCES}

Altmann, G. T. M. (1988). Ambiguity, parsing strategies, and computational models. Language \& Cognitive Processes, 3, 73-9.7.

altmann, G. T. M., Garnham, A., \& Dennis, Y. (1992). Avoiding the garden path: Eye movements in context. Journal of Memory \& Language, 31, 685-712.

altmann, G. T. M., Garnham, A., \& Henstra, J. A. (1994). Effects of syntax in human sentence parsing: Evidence against a structurebased proposal mechanism. Journal of Experimental Psychology: Learning, Memory, \& Cognition, 20, 209-216.

Altmann, G. T. M., \& Steedman, M. J. (1988). Interaction with context during human sentence processing. Cognition, 30, 191-238.

Britt, M. A., Perfetti, C. A., Garrod, S., \& Rayner, K. (1992). Parsing in discourse: Context effects and their limits. Journal of Memory \& Language, 31, 293-314.

Crain, S., \& Steedman, M. J. (1985). On not being led up the garden path: The use of context by the psychological parser. In D. R. Dowty, L. Karttunen, \& A. M. Zwicky (Eds.), Natural language parsing: Psychological, computational, and theoretical perspectives (pp. 320358). Cambridge: Cambridge University Press.

Ferreira, F., \& Clifton, C. (1986). The independence of syntactic processing. Journal of Memory \& Language, 25, 348-368.

FRAZIER, L. (1979). On comprehending sentences: Syntactic parsing strategies. Bloomington, IN: Indiana University Linguistics Club.

FrAZIER, L., \& RAYNER, K. (1982). Making and correcting errors during sentence comprehension: Eye movements in the analysis of structurally ambiguous sentences. Cognitive Psychology, 14, 178-210.

JUST, M. A., \& CARPENTER, P. A. (1992). A capacity theory of comprehension: Individual differences in working memory. Psychological Review, 99, 122-149.

Rayner, K., Carlson, M., \& Frazier, L. (1983). The interaction of syntax and semantics during sentence processing: Eye movements in the analysis of semantically biased sentences. Journal of Verbal Learning \& Verbal Behavior, 22, 358-374.

Rayner, K., Garrod, S., \& Perfetti, C. A. (1992). Discourse influences during parsing are delayed. Cognition, 45, 109-139.

Rayner, K., \& SERENo, S. C. (1994). Regressive eye movements and sentence parsing: On the use of regression-contingent analyses. Memory \& Cognition, 22, 281-285.

Rayner, K., Sereno, S. C., Morris, R. K., Schmauder, A. R., \& Clifton, C. (1989). Eye movements and on-line language comprehension processes. Language \& Cognitive Processes, 4, SI21-50.

Steedman, M. J., \& AltmanN, G. T. M. (1989). Ambiguity in context: A reply. Language \& Cognitive Processes, 4, SI105-122.

\section{NOTES}

1. Subsequent references to first-pass reading times may drop the "per character." As will become apparent, the fact that these were always per character reading times is significant. Unless explicitly indicated, all reading times referred to in the main text are first-pass reading times.

2. Per character reading times on regression trials were lower in Altmann et al.'s (1992) study than on no-regression trials. This could be because the eyes spend less time in the region prior to a regression than when they read the entire region without regressing out, and because the per character calculation results in artificially lowered reading times on regression trials (see below). If there tend to be more trials with regressions when there is a garden path, eliminating these trials will increase per character reading times more than will eliminating them from the control (unambiguous, or minimally attached) conditions. Hence the more pronounced garden path effect.

3. Although Rayner et al. (1992) found no effect of context on firstpass reading times, they did find an effect on total-pass reading times. They interpret this to mean that antecedent focus (the variable they ma- 
nipulated in that study) facilitates reanalysis following a garden paththe more focused the antecedent, the easier is recovery. This could well be true, but it is confounded in their study with the fact that the content of the nominal modifier (equivalent to the relative clause that he'd risked his life for in the earlier example) appears either closer to, or farther from, the target sentence. So not only did the distance of the critical noun phase (cf. the woman) vary, in terms of textual distance between the introduction of the referent and the target sentence, but so did the distance of the modifying information vary. When the garden path is encountered, the processor has to reanalyze the ambiguous sequence as a nominal modifier, and in so doing, establish a match between the content of the modifier and the equivalent information in the context (cf. Altmann, 1988). If this matching process is sensitive to recency (cf. distance), this would account, in part, for the particular pattern of total-pass reading times that were observed in that study.

4. It would be interesting to know what would happen if one were to reanalyze, for instance, the data reported by Britt, Perfetti, Garrod, and Rayner (1992), who found evidence of contextual influences on initial parsing decisions in certain cases (involving certain kinds of prepositional modifier) but not in others (involving reduced relative clauses).

5 . Strictly speaking, the number won't be arbitrary, but will be larger than that required to accurately reflect the number of characters actually read. Consequently, per character reading times on the affected trials will give an underestimation of the actual reading time per character.

6. I acknowledge that regression-contingent reading times per character, as reported in Altmann et al. (1992), are not free of this last problem concerning regressive movements within a region.

7. Rayner et al. (1989) suggest that summed gaze durations may therefore be more appropriate.

(Manuscript received March 17, 1993; revision accepted for publication June 4, 1993.) 